\title{
To come home after a stroke: patients' early experiences of health and recovery in their home settings
}

\author{
Elizabeth Marcheschi ${ }^{1}$, Lena Von Koch ${ }^{2}$, Hélène Pessah-Rasmussen ${ }^{3}$, Marie Elf 4 \\ 1 Chalmers University, Gothenburg, Sweden \\ 2 Department of neurobiology, care sciences and society, Stockholm, Karolinska Institutet, Sweden \\ 3 Faculty of Medicine, Lund, Lund University, Sweden \\ 4 School of Education, Health and Social Studies, Dalarna, Dalarna University, Sweden
}

The findings suggest an association between the perceived quality of the physical home environment and health outcomes after stroke that needs further investigation.

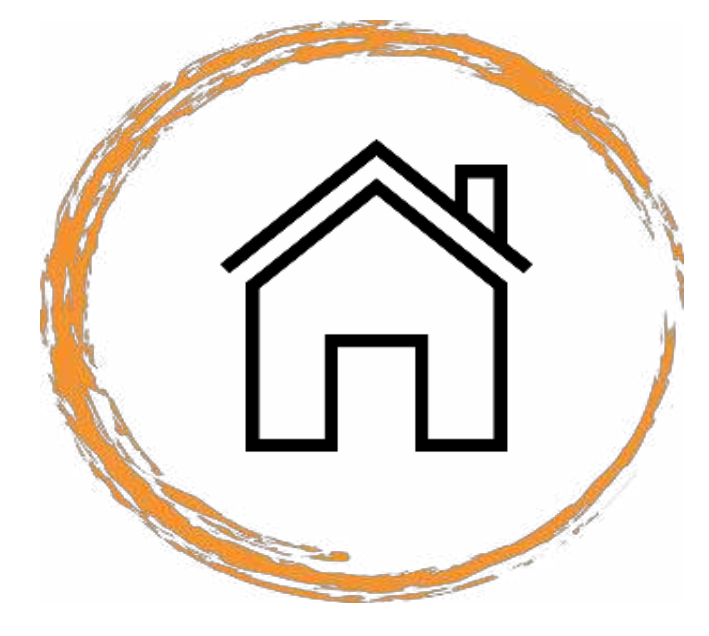

Background: Health services for stroke patients are increasingly performed within home settings, however, little knowledge is available with regard to how the physical home environment might affect health outcomes.

Study design: A cross-sectional study on people with stroke $(\mathrm{N}=16)$ was conducted with a face-to-face interview. Data was collected about, the perceived quality of physical home settings, self-efficacy, wellbeing and on facilitator and barriers.

Results: Participants who perceived their physical home environment to be of higher quality reported higher levels of self-efficacy $(U=16.0, p=.05)$ and wellbeing $(\mathrm{U}=8.0, \mathrm{p}=.005)$ than those who rated their home to be of lower quality.
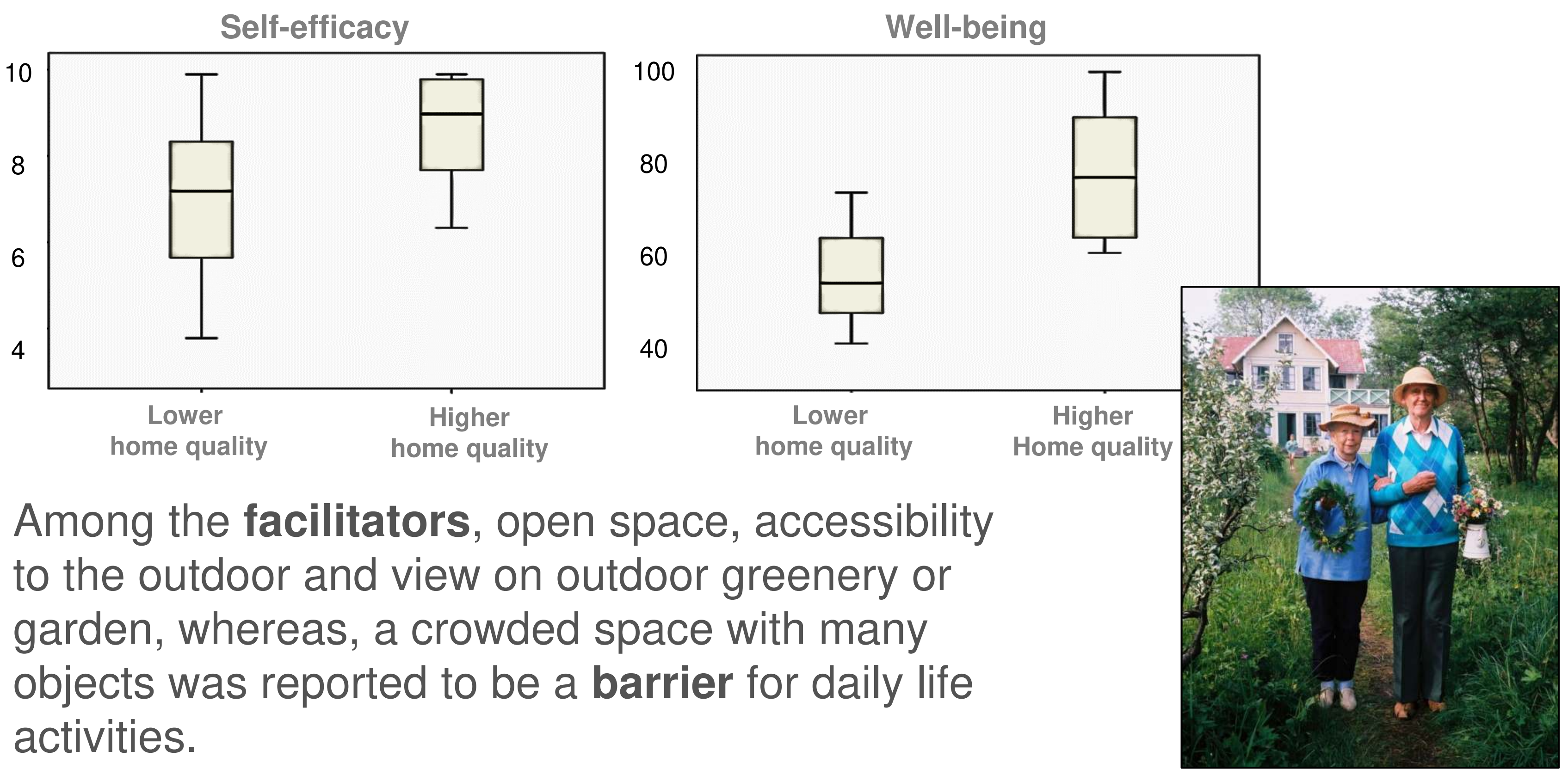

Among the facilitators, open space, accessibility to the outdoor and view on outdoor greenery or garden, whereas, a crowded space with many objects was reported to be a barrier for daily life activities. 